\title{
BMJ Open Variation in survival after out-of- hospital cardiac arrest between receiving hospitals in Japan: an observational study
}

Satoshi Koyama (D) , ${ }^{1,2}$ Koichiro Gibo, ${ }^{1}$ Yutaka Yamaguchi, ${ }^{1,2}$ Masashi Okubo ${ }^{3}$

To cite: Koyama S, Gibo K, Yamaguchi Y, et al. Variation in survival after out-of-hospital cardiac arrest between receiving hospitals in Japan: an observational study. BMJ Open 2019:9:e033919. doi:10.1136/ bmjopen-2019-033919

- Prepublication history for this paper is available online. To view these files, please visit the journal online (http://dx.doi org/10.1136/bmjopen-2019033919).

Received 28 August 2019 Revised 03 October 2019 Accepted 24 October 2019

Check for updates

(c) Author(s) (or their employer(s)) 2019. Re-use permitted under CC BY-NC. No commercial re-use. See rights and permissions. Published by BMJ.

${ }^{1}$ Department of Emergency Medicine, Okinawa Prefectural Chubu Hospital, Uruma, Okinawa, Japan

${ }^{2}$ Department of Clinical Research and Education, University of the Ryukyus, Nakagami-gun, Okinawa, Japan ${ }^{3}$ Department of Emergency Medicine, University of Pittsburgh School of Medicine, Pittsburgh, Pennsylvania, USA

Correspondence to

Dr Satoshi Koyama;

satoshi.koyama222@gmail.com

\section{ABSTRACT}

Objectives Patient outcomes after out-of-hospital cardiac arrest $(\mathrm{OHCA})$ varies at multilevel (geographical regions, emergency medical service agencies and receiving hospitals) in the USA. However, it remains unclear whether there is a variation in patient outcomes after $\mathrm{OHCA}$ between relevant units of the healthcare system such as receiving hospitals in Japan. Therefore, we aimed to quantify the variation in patient outcomes after $\mathrm{OHCA}$ between receiving hospitals in Japan.

Design Secondary analysis of the prospective multicentre OHCA registry.

Setting The Japan Association for Acute Medicine OHCA Registry, a prospective multicentre OHCA registry, including 73 medical institutions in Japan.

Participants 9303 adults ( $\geq 18$ years old) with OHCA of medical origin, treated at 67 hospitals from June 2014 to December 2015.

Primary and secondary outcome measures The primary outcome was 1-month survival after $\mathrm{OHCA}$. The secondary outcome was favourable functional status at 1 month, defined as cerebral performance category scale 1 or 2 . We constructed a series of multivariable hierarchical logistic regression models predicting outcomes, accounting for patient-level variables and clustering of patients within hospitals. We evaluated the adjusted 1-month survival and functional outcome for each hospital, ranked hospitals for each outcome and calculated median ORs (MORs) to quantify the between-hospital variation in outcomes.

Results The prevalence of 1-month survival after OHCA was $7.1 \%$ (663/9303) and that of favourable functional outcome was 3.6\% (331/9303). After adjustment for patient-level factors, we observed variations in 1-month survival (range, 1.6\%-13.8\%; adjusted MOR 1.34; $95 \% \mathrm{Cl}$ 1.16 to 1.67 ) and favourable functional outcome (range, $0.7 \%-7.3 \%$; adjusted MOR 1.53; $95 \% \mathrm{Cl} 1.10$ to 2.24 ) between hospitals.

Conclusions We found substantial variations in patient outcomes after OHCA within a large group of hospitals in Japan, despite adjustment for patient factors that are known to be associated with different outcomes.

\section{INTRODUCTION}

Out-of-hospital cardiac arrest (OHCA) is one of the most important public health problems worldwide. Overall, 356000 individuals in the
Strengths and limitations of this study

- We constructed a series of multivariable hierarchical logistic regression models predicting outcomes and calculated the median $\mathrm{OR}$ to quantify the variation in patient outcomes at hospital level with adjusting for patient-level factors.

- We observed substantial variations in patient outcomes, median differences of $34 \%$ in the odds of 1-month survival and $53 \%$ in the odds of favourable functional outcome at 1 month for any two otherwise similar patients who were treated by any two randomly selected hospitals

- The Japan Association for Acute Medicine OHCA Registry was combined with the All-Japan Utstein Registry. $10.9 \%$ of patients who were unable to be combined were excluded from the analysis as missing data, resulting in potential risk of selection bias.

USA and 127000 in Japan annually develop OHCA with high mortality. ${ }^{12}$ During the last decades, prior studies revealed factors that impact patient outcomes after OHCA, such as prehospital factors (quality of cardiopulmonary resuscitation (CPR) and early defibrillation), ${ }^{3-6}$ patient factors (initial rhythm, age and origin of cardiac arrest) ${ }^{7}$ and hospital factors (post-resuscitation care options, eg, targeted temperature management and early coronary angiography). ${ }^{8-10}$ These findings enhanced resuscitation guidelines and survival after OHCA has been improving in some communities. $^{1112}$

Prior studies also showed that patient outcomes after OHCA significantly varied at multiple levels: between geographical regions, ${ }^{13} 14$ emergency medical service (EMS) agencies $^{15}$ and receiving hospitals ${ }^{16-19}$ in the USA, suggesting important disparities in healthcare provision. We recently reported substantial variation in functional outcome after OHCA in geographical regions in Japan between all 47 prefectures. $^{20}$ However, it remains unclear whether there is a variation in 
patient outcomes after OHCA between relevant units in the healthcare system, such as receiving hospitals. Detecting existing differences in health outcomes is the first step in the research of healthcare disparities, enabling to further understanding and reduction of the observed disparities. ${ }^{21}$

Our objective is to quantify the extent of betweenhospital variation in survival and functional outcome after OHCA in Japan, accounting for patient demographics, cardiac arrest characteristics and prehospital interventions as well as clustering of patients within receiving hospitals.

\section{METHODS}

\section{Study design and setting}

We performed a secondary analysis of the Japan Association for Acute Medicine OHCA Registry (JAAM-OHCA Registry). The methodology of the JAAM-OHCA Registry was previously reported elsewhere. ${ }^{22}$ Briefly, the JAAM-OHCA Registry is a prospective multicentre OHCA registry with voluntary participation of 73 medical institutions in Japan. The registry included all patients with OHCA, defined as initiation of resuscitation attempts with shock delivery by an external defibrillator (by a layperson or EMS personnel) or chest compressions by EMS personnel, who were then transported to participating institutions between June 2014 and December 2015 (the most recent publicly available data at the time of the analysis). In Japan, all patients with OHCA who receive resuscitation by EMS personnel are transported to medical institutions regardless of the presence of return of spontaneous circulation (ROSC). The choice of transporting hospital is at the discretion of the EMS providers. The registry excluded OHCA patients who did not receive CPR at the participating institutions (ie, when resuscitative efforts were immediately terminated on hospital arrival), or for whom participation in the registry was refused either personally or by family members. OHCA patients who were transferred to the participating institutions from another hospital were also excluded. The registry is also combined with the All-Japan Utstein Registry of the Fire and Disaster Management Agency (FDMA), a prospective, nationwide, population-based registry of OHCA cases based on the Utstein style in Japan.

\section{Receiving hospital setting}

The JAAM-OHCA Registry is a voluntary registry of participating hospitals with emergency departments, which includes critical care medical centres (CCMCs) certified by the Japanese Ministry of Health, Labour and Welfare that can accept emergency and severely ill patients transported by ambulance, including OHCA patients. To be licensed as a CCMC, a hospital should have $\geq 20$ beds and an intensive care unit and be able to provide highly specialised treatments such as extracorporeal resuscitation, targeted temperature management and percutaneous coronary intervention (PCI), 24hours a day. CCMCs as well as nonCCMCs with an emergency care department can participate in this registry. The number of institutions participating to the JAAM-OHCA Registry was 73 in $2015 .{ }^{22}$

\section{Study population}

We included adults ( $\geq 18$ years old) with EMS-treated OHCA of medical origin who were registered with the JAAM-OHCA Registry. ${ }^{23}$ We excluded patients with missing data for important patient-level covariates, patients whose initial rhythm code was 'others', patients with OHCA of non-medical origin (trauma, toxicity, drowning, traffic injury, hypothermia, anaphylaxis and others), and patients treated by hospitals that treated 10 or fewer OHCA cases annually in this dataset. ${ }^{1517}$

\section{Study variables}

Study variables included in risk-adjusted models were (1) patient demographics: age (continuous) and sex (male or female); (2) cardiac arrest event characteristics: cause of arrest (cardiac, non-cardiac), initial rhythm (ventricular fibrillation (VF)/ventricular tachycardia (VT) or pulseless electrical activity (PEA)/asystole), witnessed collapse (none, a bystander or EMS personnel); (3) layperson interventions: bystander CPR (presence or absence), dispatcher CPR instruction (presence or absence) and shock delivery with public automated external defibrillator (AED) (presence or absence); (4) EMS interventions: prehospital defibrillation (presence or absence), prehospital advanced airway placement with tracheal intubation or supraglottic airway device (yes or no), and prehospital epinephrine administration (presence or absence); (5) EMS time variables: the interval from the first telephone call to CPR initiation by EMS providers and from CPR initiation by EMS providers to hospital arrival (measured as continuous variables). These covariates were chosen a priori based on their known associations with survival from prior studies of OHCA, biologic plausibility and adequate ascertainment. ${ }^{3-7}$

\section{Outcome measure}

The primary outcome was 1-month survival after OHCA. The secondary outcome was functional status at 1 month after OHCA occurrence using the Glasgow-Pittsburgh cerebral performance category (CPC) scale (category 1, good cerebral performance; 2, moderate cerebral disability; 3 , severe cerebral disability; 4 , coma or vegetative state; 5 , death/brain death). Favourable functional outcome was defined as a CPC of 1 or $2 .{ }^{24} 25$

\section{Statistical analysis}

We first described the unadjusted rate of 1-month survival after OHCA within each hospital. We ranked and grouped hospitals into quartiles according to their unadjusted survival rates and reported differences in patient-level characteristics by quartiles. We described the variability in unadjusted 1-month survival and favourable functional outcome between receiving hospitals.

We then constructed a series of multivariable hierarchical logistic regression models to predict 1-month survival and favourable functional outcome, based on previously described variables. These models accounted for potential 
confounders and clustering of patients within each hospital. We treated hospitals as a random effect and nested individual patients within each hospital, whereas patient-level variables were modelled as fixed effects. From these models, we calculated adjusted 1-month survival and favourable functional outcome for each hospital and ranked hospitals for each outcome. We also calculated median ORs (MORs) and $95 \% \mathrm{CI}$ to quantify the between-hospital variation in outcomes. ${ }^{26}{ }^{27}$ We derived MORs from the variance estimate of the random intercept in the hierarchical regression models by Bayesian method. ${ }^{26}{ }^{27}$ Conceptually, the MORs represent the relative odds of outcome for two patients with similar characteristics between two different, randomly selected hospitals. ${ }^{26}{ }^{27}$ For example, an MOR of 2.0 indicates a median twofold difference in the odds of outcome for similar individuals treated by two different, randomly selected hospitals. Subgroup analyses of 1-month survival and favourable functional outcome were conducted to confirm the consistency of between-hospital variation within each subgroup. Subgroups were defined by initial rhythm: those with a shockable rhythm (VF, VT and shocked by an AED) versus non-shockable rhythm (PEA, asystole and not shocked by an AED); witnessed collapse: witnessed (a bystander or EMS personnel) versus non-witnessed; the presence of ROSC prior to hospital arrival (field ROSC): field ROSC versus non-field ROSC. Field ROSC was defined as palpable pulse prior to hospital arrival at least once.

We used R V.3.2.4 (The R Foundation for Statistical Computing, Vienna, Austria) and Stata V.12 (StataCorp, College Station, Texas, USA) for our analyses.

\section{Patient and public involvement}

In this study, patients and public were not involved. We performed a secondary analysis of the data of the JAAMOHCA Registry as described above.

\section{RESULTS}

The patient flow diagram is seen in figure 1. From June 2014 to December 2015, 13469 patients with EMS-treated OHCA were registered with the JAAM Registry. Of these, 9303 patients with EMS-treated OHCA of medical origin who were treated at 67 hospitals were eligible for our analyses after excluding 3850 patients who met exclusion criteria. Patients with missing data included 1467 patients who were not included in the Utstein data of FDMA and 14 patients in whom prehospital epinephrine administration was unknown. The prevalence of ROSC after OHCA was $12.2 \%$ (1137/9303), 1-month survival after OHCA was $7.1 \%$ (663/9303), and favourable functional outcome was $3.6 \%(331 / 9303)$.

Patient demographics are summarised in table 1 . When categorising hospitals into quartiles based on the rate of patient survival, patients treated by the highest quartile hospitals, compared with those at the lowest quartile hospitals, tended to be younger. In cardiac arrest event characteristics, cardiac cause, non-shockable initial rhythms (PEA/asystole) and unwitnessed arrest were observed less

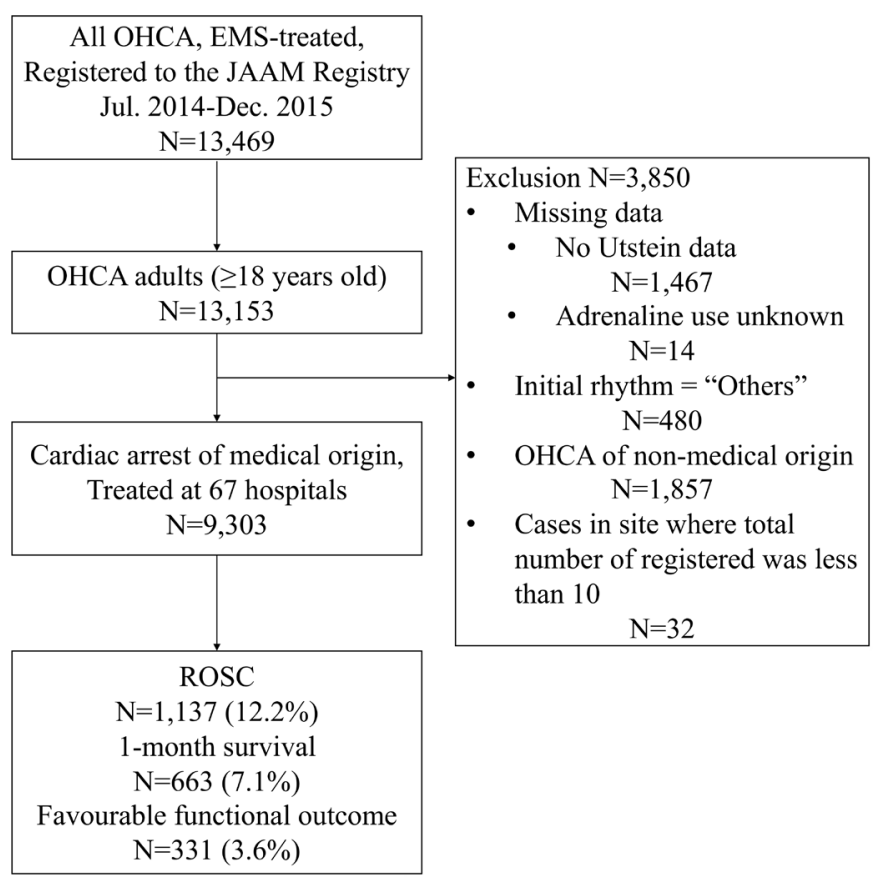

Figure 1 Flow diagram for patient selection. OHCA, outof-hospital cardiac arrest; EMS, emergency medical service; JAAM, Japan Association for Acute Medicine; ROSC, return of spontaneous circulation.

frequently in the patients treated by the highest quartile hospitals, compared with that in the patients treated by the lowest quartile hospitals. For layperson interventions, patients treated by the highest quartile hospitals, compared with those at the lowest quartile hospitals, tended to receive bystander CPR, dispatcher CPR instruction and shock delivery with public-access AEDs more often. For EMS interventions, patients treated by the highest quartile hospitals, compared with those at the lowest quartile hospitals, tended to receive defibrillation by EMS providers, advanced airway management and epinephrine administration more often.

After calculating adjusted 1-month survival and favourable functional outcome for each hospital, we ranked hospitals and constructed a caterpillar plot (figure 2). The adjusted rate of 1-month survival after OHCA in each hospital ranged from $1.6 \%$ to $13.8 \%$ (figure $2 \mathrm{~A}$ ). The adjusted rate of favourable functional outcome ranged from $0.7 \%$ to $7.3 \%$ (figure $2 \mathrm{~B}$ ).

The primary and secondary outcomes are shown in table 2. The unadjusted MOR for 1-month survival after OHCA was 1.49 (95\% CI 1.26 to 1.91). After adjustment for patient-level factors, the MOR for 1-month survival after OHCA slightly decreased to 1.34 (95\% CI 1.16 to 1.67 ). The unadjusted MOR for favourable functional outcome was 1.91 (95\% CI 1.40 to 3.00). After adjustment for patientlevel factors, the MOR for favourable functional outcome slightly decreased to 1.53 (95\% CI 1.20 to 2.24 ).

The adjusted MORs for survival and functional outcome among the subgroups stratified by initial rhythm, witnessed collapse and the presence of ROSC prior to the hospital arrival are seen in table 3. 


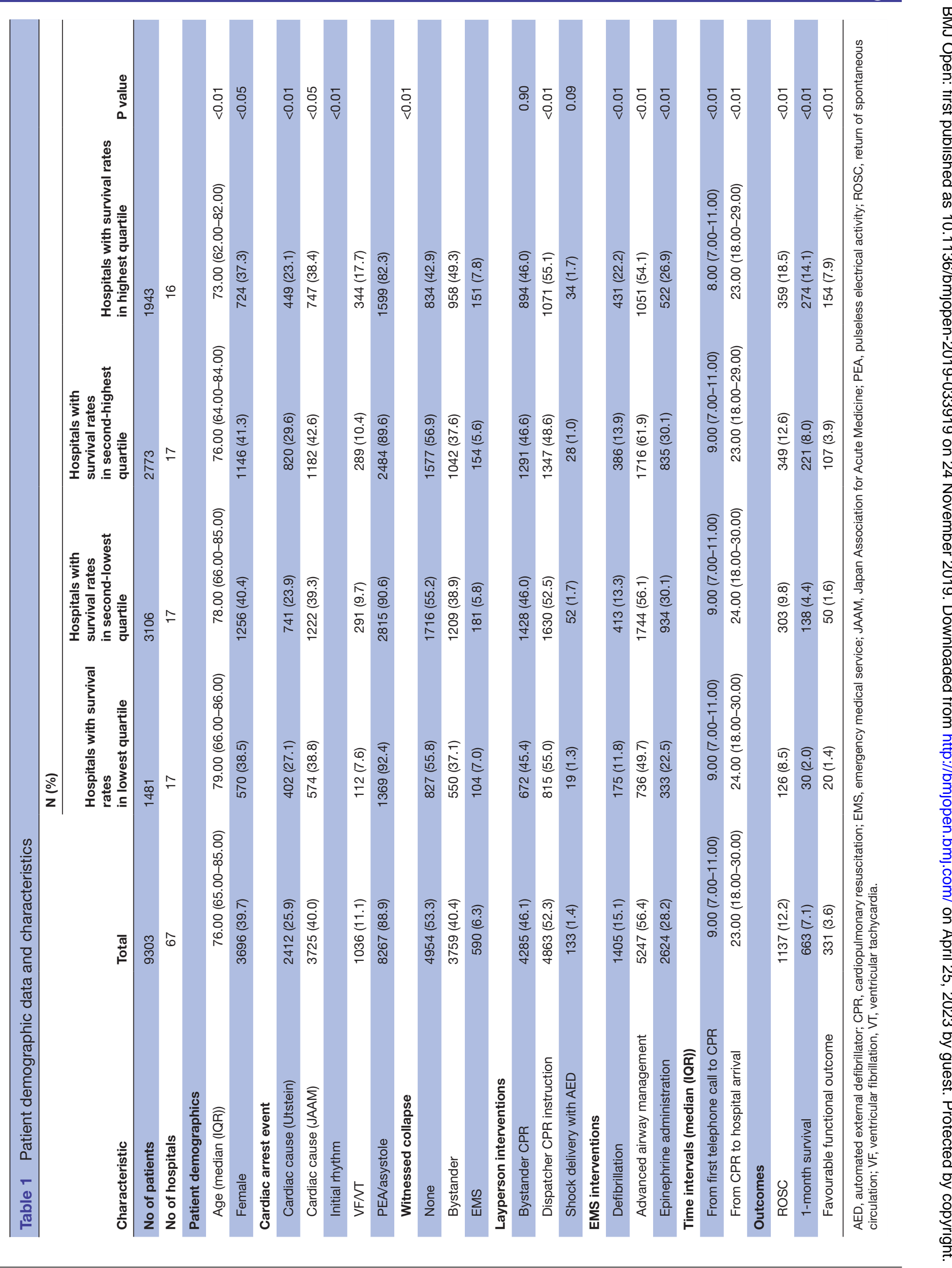

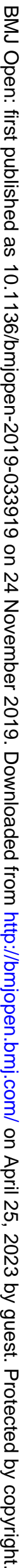


A

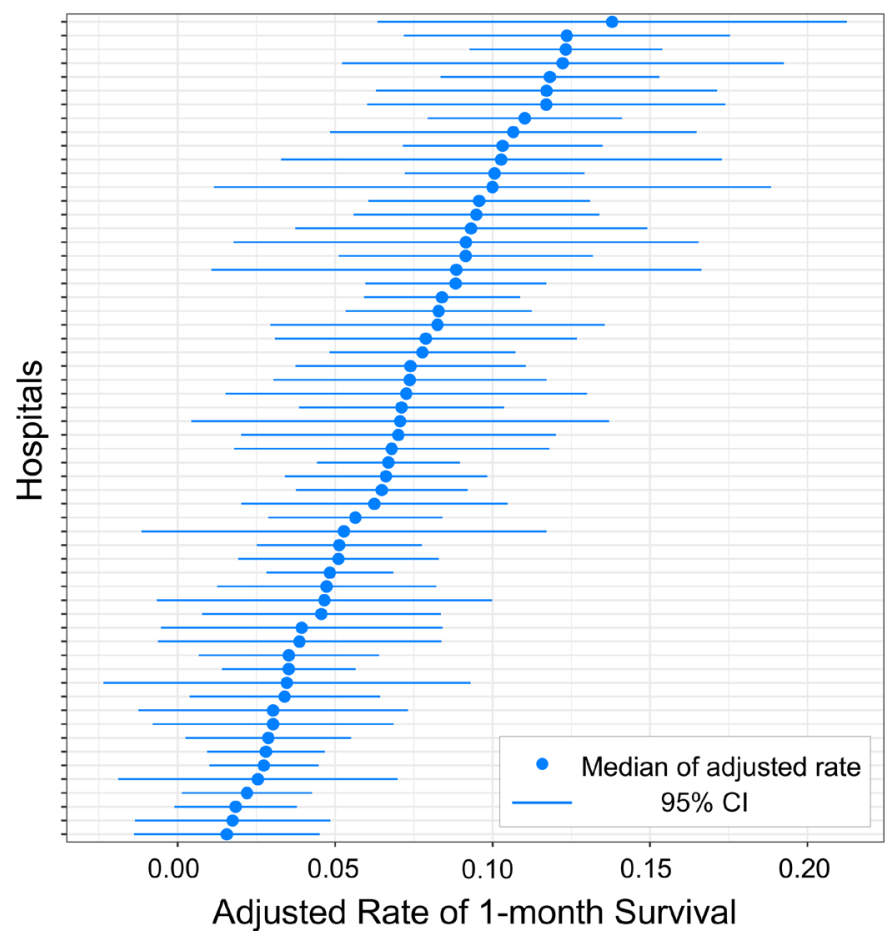

B

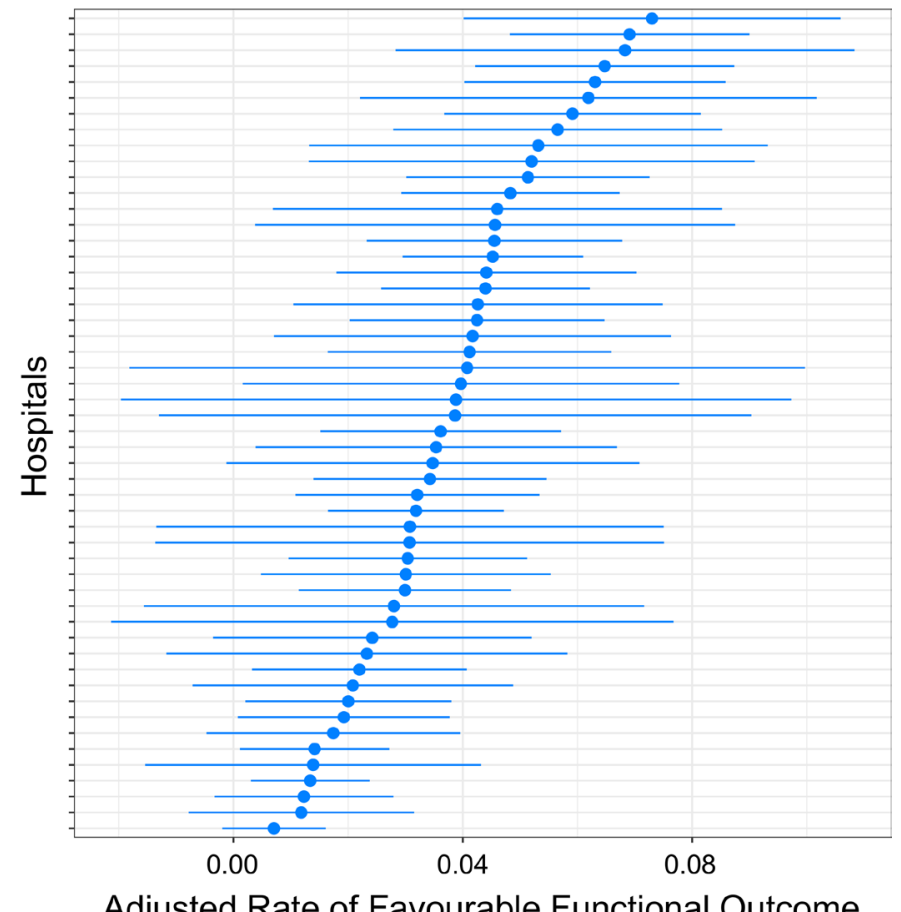

Figure 2 Caterpillar plot for adjusted rate of 1-month survival (A) and favourable functional outcome (B) among OHCA patients within each hospital. Adjusted for age, sex, cause of arrest (cardiac or non-cardiac), initial rhythm (VF/VT or PEA asystole), witnessed collapse (none, a bystander or EMS personnel), bystander CPR (presence or absence), dispatcher CPR instruction (presence or absence), shock delivery with public AED (presence or absence), prehospital defibrillation (presence or absence), prehospital advanced airway placement with tracheal intubation or supraglottic airway device (yes or no), prehospital epinephrine administration (presence or absence), the interval from the first telephone call to CPR initiation by EMS providers and that from CPR initiation by EMS providers to hospital arrival. AED, automated external defibrillator; CPR, cardiopulmonary resuscitation; EMS, emergency medical service; OHCA, out-of-hospital cardiac arrest; PEA, pulseless electrical activity; VF, ventricular fibrillation, VT, ventricular tachycardia.

\section{DISCUSSION}

In this analysis of a large prospective registry of OHCA in Japan, we found substantial differences in 1-month survival and favourable functional outcome between the 67 receiving hospitals. These differences persisted after adjustment for known patient and prehospital factors. We observed median differences of $34 \%$ in the odds of survival and $53 \%$ in the odds of favourable functional outcome at 1 month for any two otherwise similar patients who were treated by any two randomly selected hospitals. Our data suggest that the between-hospital variation resulted from unadjusted patient, EMS, hospital and/or community characteristics.

Prior studies reported that factors at multiple levels (eg, patient, EMS, hospital and geographical region level) were associated with patient outcomes after OHCA. ${ }^{3-10}$ Mumma et al reported significant variation in good neurological recovery following OHCA between 128 hospitals where PCI is available 24 hours $/ 7$ days in California, USA. Multivariable hierarchical logistic regression models were constructed (adjusted rates of good neurological recovery, 18\% to 39\%; $\mathrm{p}<0.001) .{ }^{28}$ Our present study corroborated this prior study and confirmed the between-hospital variation in survival and functional outcome after OHCA in different healthcare settings. The between-hospital variation might result from the hospital characteristics that prior studies reported, such as OHCA case volume, ${ }^{17-19} 2930$ and cath-lab availability (24/7 PCI or limited PCI capability).$^{30-32}$ However, in Mumma's study, the between-hospital variation in good neurological recovery following OHCA existed even though hospitals with similar characteristics (24/7 PCI centre) were incorporated. ${ }^{28}$ These results suggest that there were unmeasured hospital characteristics causing the between-hospital variation. On the other hand, Nallamothu et al evaluated resuscitation teams at hospitals with high in-hospital cardiac arrest survival with a qualitative study and discovered four broad themes that distinguish top-performing hospitals: team design, team composition and roles, communication and leadership, and training and educational efforts. ${ }^{33}$ This may suggest that resuscitation team characteristics and organisation, both prehospital and in-hospital, contribute to the variation in patient outcomes after OHCA. Further research is needed to identify characteristics of high-performing hospitals in OHCA and should be targeted in future interventions to improve care after OHCA.

In our subgroup analyses, there were significant betweenhospital variations among all subgroups in 1-month survival and favourable functional outcome. We observed larger between-hospital variations in survival and favourable 
Table 2 Between-hospital variation in 1-month survival and favourable functional outcome after OHCA

\section{Median OR $(95 \% \mathrm{Cl})$}

\begin{tabular}{ll}
\hline 1-month survival & \\
\hline Unadjusted & $1.49(1.26$ to 1.91$)$ \\
$\begin{array}{l}\text { Adjusted for select } \\
\text { variables }\end{array}$ & $1.34(1.16$ to 1.67$)$ \\
\end{tabular}

\section{Favourable functional} outcome

$\begin{array}{ll}\text { Unadjusted } & 1.91(1.40 \text { to } 3.00) \\ \begin{array}{l}\text { Adjusted for select } \\ \text { variables* }\end{array} & 1.53(1.20 \text { to } 2.24)\end{array}$

*Adjusted for age, sex, cause of arrest (cardiac or non-cardiac), initial rhythm (VF/VT or PEA/asystole), witnessed collapse (none, a bystander or EMS personnel), bystander CPR (presence or absence), dispatcher CPR instruction (presence or absence), shock delivery with public AED (presence or absence), prehospital defibrillation (presence or absence), prehospital advanced airway placement with tracheal intubation or supraglottic airway device (yes or no), prehospital epinephrine administration (presence or absence), the interval from the first telephone call to CPR initiation by EMS providers and that from CPR initiation by EMS providers to hospital arrival.

AED, automated external defibrillator; CPR, cardiopulmonary resuscitation; EMS, emergency medical service; OHCA, outof-hospital cardiac arrest; PEA, pulseless electrical activity; VF, ventricular fibrillation; VT, ventricular tachycardia.

functional outcome among shockable and witnessed groups, compared with non-shockable and unwitnessed groups. This might suggest that hospital factors such as post-resuscitation care at hospital level (eg, PCI and targeted temperature management) are determinants of patient outcomes among patients with favourable features. On the other hand, those without field ROSC had a larger variation in survival, compared with those with field ROSC. This suggests that performance of CPR after hospital arrival contributed to survival in those without field ROSC.

Our study has several limitations. First, we cannot determine which unmeasured factors explain the observed differences in outcomes. These unmeasured factors include patient comorbidity, ${ }^{34}$ experience and training of treating EMS personnel, ${ }^{34}$ post-resuscitation practice ${ }^{8-10}$ and neighbourhood factors. ${ }^{35}$ Second, our inference may not be fully generalisable to other healthcare settings. There are 288 tertiary hospitals to which OHCA patients are transported in Japan and 73 hospitals were registered in this dataset. ${ }^{22}$ There may be selection bias due to the characteristics of the registered hospitals, for example, hospitals in the registry may have emergency departments with higher motivation for resuscitation. Third, the JAAM-OHCA registry excluded cases wherein resuscitative efforts were immediately terminated on hospital arrival. The decision to terminate resuscitation could have varied between hospitals, potentially leading to selection bias. However, in subgroup analyses, we observed persisting large variation in outcomes among those with known favourable features such as shockable initial rhythm, witnessed arrest
Table 3 Adjusted median ORs for 1-month survival and favourable functional outcome of OHCA patients stratified according to pre-specified subgroups

Adjusted median OR* $(95 \% \mathrm{Cl})$

Favourable functional

1-month survival outcome

\begin{tabular}{|c|c|c|}
\hline \multicolumn{3}{|l|}{ Initial rhythm } \\
\hline Shockable & 1.47 (1.19 to 2.03$)$ & 1.55 (1.14 to 2.53$)$ \\
\hline Non-shockable & $1.32(1.09$ to 1.81$)$ & $1.32(1.02$ to 2.19$)$ \\
\hline \multicolumn{3}{|l|}{ Witnessed collapse } \\
\hline Witnessed & $1.39(1.16$ to 1.82$)$ & $1.67(1.25$ to 2.69$)$ \\
\hline Non-witnessed & $1.22(1.00$ to 1.87$)$ & $1.18(1.00$ to 2.29$)$ \\
\hline \multicolumn{3}{|c|}{$\begin{array}{l}\text { Presence of ROSC } \\
\text { prior to the hospital } \\
\text { arrival }\end{array}$} \\
\hline Field ROSC & $1.12(1.01$ to 1.40$)$ & 1.35 (1.02 to 2.22$)$ \\
\hline Non-field ROSC & 1.35 (1.08 to 1.90$)$ & $1.12(1.00$ to 1.62$)$ \\
\hline
\end{tabular}

*Adjusted for age, sex, cause of arrest (cardiac or non-cardiac), initial rhythm (VF/VT or PEA/asystole), witnessed collapse (none, a bystander or EMS personnel), bystander CPR (presence or absence), dispatcher CPR instruction (presence or absence), shock delivery with public AED (presence or absence), prehospital defibrillation (presence or absence), prehospital advanced airway placement with tracheal intubation or supraglottic airway device (yes or no), prehospital epinephrine administration (presence or absence), the interval from the first telephone call to CPR initiation by EMS providers and that from CPR initiation by EMS providers to hospital arrival.

AED, automated external defibrillator; CPR, cardiopulmonary resuscitation; OHCA, out-of-hospital cardiac arrest; PEA, pulseless electrical activity; ROSC, return of spontaneous circulation; VF, ventricular fibrillation; VT, ventricular tachycardia.

and field ROSC, supporting the robustness of our findings, because those with favourable features would have been less likely to impact selection bias. Fourth, as the data collection period was between 2014 and 2015, our results may not reflect current variation in patient outcomes. Lastly, there are 1467 patients (10.9\% of all registered patients) who could not be combined with the data of All-Japan Utstein Registry. We excluded these patients from the analysis as missing data, resulting in a risk of selection bias.

\section{CONCLUSIONS}

In this secondary analysis of a large prospective OHCA registry in Japan, patient outcomes after OHCA varied substantially between hospitals, despite adjustment for known factors associated with different outcomes. Further research is required to identify modifiable factors that contribute to this observed variation to improve patient outcomes after OHCA.

Acknowledgements We are deeply indebted to all members and institutions of the JAAM-OHCA Registry for their contribution. The participating institutions of the JAAM-OHCA Registry are listed online (http://www.jaamohca-web.com/list/). We also acknowledge the assistance of Clifton W Callaway, MD PhD, Professor of 
Emergency Medicine at the University of Pittsburgh, with expert advice. We would like to thank Editage (http://www.editage.jp) for English language editing.

Contributors MO contributed in the study concept and design. SK, KG and YY were involved in the acquisition of data. SK, KG, YY and MO participated in the analysis and interpretation of data. SK and MO drafted the manuscript. SK, KG, YY and MO performed the critical revision of the manuscript for important intellectual content. KG performed the statistical analysis.

Funding The authors have not declared a specific grant for this research from any funding agency in the public, commercial or not-for-profit sectors.

\section{Competing interests None declared.}

\section{Patient consent for publication Obtained.}

Ethics approval Investigators acquired data under a waiver of informed consent for a minimal risk intervention with institutional review board approval at each co-ordinating site. The JAAM data co-ordinating centre created the final database from de-identified data transmitted from participating sites. The institutional review board at Okinawa Chubu Hospital approved this secondary analysis of de-identified data.

\section{Provenance and peer review Not commissioned; externally peer reviewed.}

Data availability statement Data may be obtained from a third party and are not publicly available.

Open access This is an open access article distributed in accordance with the Creative Commons Attribution Non Commercial (CC BY-NC 4.0) license, which permits others to distribute, remix, adapt, build upon this work non-commercially, and license their derivative works on different terms, provided the original work is properly cited, appropriate credit is given, any changes made indicated, and the use is non-commercial. See: http://creativecommons.org/licenses/by-nc/4.0/.

\section{ORCID iD}

Satoshi Koyama http://orcid.org/0000-0002-9637-8440

\section{REFERENCES}

1 Benjamin EJ, Blaha MJ, Chiuve SE, et al. Heart Disease and Stroke Statistics - 2017 Update: a report from the American Heart Association. Circulation 2017;135:e146-603.

2 The Fire and Disaster Management Agency of Japan. Current status of emergency and rescue in Heisei 30th 2018. Available: https:// www.fdma.go.jp/publication/rescue/items/kkkg_h30_01_kyukyu.pdf

3 Soo LH, Gray D, Young T, et al. Influence of ambulance crew's length of experience on the outcome of out-of-hospital cardiac arrest. Eur Heart J 1999;20:535-40.

4 Gold LS, Eisenberg MS. The effect of paramedic experience on survival from cardiac arrest. Prehosp Emerg Care 2009;13:341-4

5 Hallstrom AP, Ornato JP, Weisfeldt M, et al. Public-access defibrillation and survival after out-of-hospital cardiac arrest. $N$ Engl J Med 2004;351:637-46.

6 Kitamura T, Kiyohara K, Sakai T, et al. Public-access defibrillation and out-of-hospital cardiac arrest in Japan. N Engl J Med 2016;375:1649-59.

7 Luo S, Zhang Y, Zhang W, et al. Prognostic significance of spontaneous shockable rhythm conversion in adult out-of-hospital cardiac arrest patients with initial non-shockable heart rhythms: a systematic review and meta-analysis. Resuscitation 2017:121:1-8.

8 Schenone AL, Cohen A, Patarroyo G, et al. Therapeutic hypothermia after cardiac arrest: a systematic review/meta-analysis exploring the impact of expanded criteria and targeted temperature. Resuscitation 2016;108:102-10.

9 Irisawa T, Matsuyama T, Iwami T, et al. The effect of different target temperatures in targeted temperature management on neurologically favorable outcome after out-of-hospital cardiac arrest: a nationwide multicenter observational study in Japan (the JAAM-OHCA registry). Resuscitation 2018;133:82-7.

10 Khera R, CarlLee S, Blevins A, et al. Early coronary angiography and survival after out-of-hospital cardiac arrest: a systematic review and meta-analysis. Open Heart 2018;5:e000809.

11 Meaney PA, Bobrow BJ, Mancini ME, et al. Cardiopulmonary resuscitation quality: [corrected] improving cardiac resuscitation outcomes both inside and outside the hospital: a consensus statement from the American Heart Association. Circulation 2013;128:417-35.

12 Travers AH, Perkins GD, Berg RA, et al. Part 3: adult basic life support and automated external defibrillation: 2015 international consensus on cardiopulmonary resuscitation and emergency cardiovascular care science with treatment recommendations. Circulation 2015;132:S51-83.

13 Nichol G, Thomas E, Callaway CW, et al. Regional variation in out-of-hospital cardiac arrest incidence and outcome. JAMA 2008;300:1423-31.

14 Girotra S, van Diepen S, Nallamothu BK, et al. Regional variation in out-of-hospital cardiac arrest survival in the United States. Circulation 2016;133:2159-68.

15 Okubo M, Schmicker RH, Wallace DJ, et al. Variation in survival after out-of-hospital cardiac arrest between emergency medical services agencies. JAMA Cardiol 2018;3:989-99.

16 Carr BG, Goyal M, Band RA, et al. A national analysis of the relationship between hospital factors and post-cardiac arrest mortality. Intensive Care Med 2009;35:505-11.

17 Carr BG, Kahn JM, Merchant RM, et al. Inter-hospital variability in post-cardiac arrest mortality. Resuscitation 2009;80:30-4.

18 Callaway CW, Schmicker R, Kampmeyer M, et al. Receiving Hospital characteristics associated with survival after out-of-hospital cardiac arrest. Resuscitation 2010;81:524-9.

19 Cudnik MT, Sasson C, Rea TD, et al. Increasing hospital volume is not associated with improved survival in out of hospital cardiac arrest of cardiac etiology. Resuscitation 2012;83:862-8.

20 Okubo M, Gibo K, Wallace DJ, et al. Regional variation in functional outcome after out-of-hospital cardiac arrest across 47 prefectures in Japan. Resuscitation 2018;124:21-8.

21 Kilbourne AM, Switzer G, Hyman K, et al. Advancing health disparities research within the health care system: a conceptual framework. Am J Public Health 2006;96:2113-21.

22 Kitamura T, Iwami T, Atsumi T, et al. The profile of Japanese Association for Acute Medicine-out-of-hospital cardiac arrest registry in 2014-2015. Acute Med Surg 2018;5:249-58.

23 Perkins GD, Jacobs IG, Nadkarni VM, et al. Cardiac arrest and cardiopulmonary resuscitation outcome reports: update of the Utstein Resuscitation Registry Templates for Out-of-Hospital Cardiac Arrest: a statement for healthcare professionals from a task force of the International Liaison Committee on Resuscitation (American Heart Association, European Resuscitation Council, Australian and New Zealand Council on Resuscitation, Heart and Stroke Foundation of Canada, InterAmerican Heart Foundation, Resuscitation Council of Southern Africa, Resuscitation Council of Asia); and the American Heart Association Emergency Cardiovascular Care Committee and the Council on Cardiopulmonary, Critical Care, Perioperative and Resuscitation. Circulation 2015;132:1286-300.

24 Jennett B, Bond M. Assessment of outcome after severe brain damage. Lancet 1975;1:480-4.

25 Edgren E, Hedstrand U, Kelsey S, et al. Assessment of neurological prognosis in comatose survivors of cardiac arrest. Lancet 1994;343:1055-9.

26 Larsen K, Merlo J. Appropriate assessment of neighborhood effects on individual health: integrating random and fixed effects in multileve logistic regression. Am J Epidemiol 2005;161:81-8.

27 Merlo J, Chaix B, Ohlsson $\mathrm{H}$, et al. A brief conceptual tutorial of multilevel analysis in social epidemiology: using measures of clustering in multilevel logistic regression to investigate contextual phenomena. J Epidemiol Community Health 2006;60:290-7.

28 Mumma BE, Wilson MD, García-Pintos MF, et al. Variation in outcomes among $24 / 7$ percutaneous coronary intervention centres for patients resuscitated from out-of-hospital cardiac arrest. Resuscitation 2019;135:14-20.

29 Schober A, Sterz F, Laggner AN, et al. Admission of out-of-hospital cardiac arrest victims to a high volume cardiac arrest center is linked to improved outcome. Resuscitation 2016;106:42-8.

30 Chocron R, Bougouin W, Beganton F, et al. Are characteristics of hospitals associated with outcome after cardiac arrest? Insights from the Great Paris registry. Resuscitation 2017;118:63-9.

31 Stub D, Schmicker RH, Anderson ML, et al. Association between hospital post-resuscitative performance and clinical outcomes after out-of-hospital cardiac arrest. Resuscitation 2015;92:45-52.

32 Hunter BR, O'Donnell DP, Kline JA, et al. Receiving hospital characteristics associated with survival in patients transported by emergency medical services after out-of-hospital cardiac arrest. Academic Emergency Medicine 2016;23:905-9.

33 Nallamothu BK, Guetterman TC, Harrod M, et al. How do resuscitation teams at top-performing hospitals for in-hospital cardiac arrest succeed? Circulation 2018;138:154-63.

34 Carew HT, Zhang W, Rea TD. Chronic health conditions and survival after out-of-hospital ventricular fibrillation cardiac arrest. Heart 2007;93:728-31.

35 Starks MA, Schmicker RH, Peterson ED, et al. Association of neighborhood demographics with out-of-hospital cardiac arrest treatment and outcomes. JAMA Cardiol 2017;2:1110-8. 